\title{
The Proteus Perspective: new capabilities of mass-filter, collision cell, multi-collector inductively- coupled mass-spectrometry (CC-MC- ICPMS/MS)
}

\author{
TIM ELLIOTT ${ }^{1}$, DAN BEVAN ${ }^{1}$, CHRISTOPHER D. \\ COATH $^{1}$, JAMIE LEWIS ${ }^{1}$, KATHRYN M.M SHAW ${ }^{1}$, TU- \\ HAN LUU $^{2}$, GRANT CRAIG ${ }^{3}$, NICHOLAS LLOYD ${ }^{4}$, \\ MARKUS PFEIFER ${ }^{3}$, HENNING WEHRS ${ }^{3}$ AND JOHANNES \\ SCHWIETERS $^{3}$ \\ ${ }^{1}$ University of Bristol \\ ${ }^{2}$ Université de Paris - IPGP - CNRS/UMR 7154 \\ ${ }^{3}$ Thermo Fisher Scientific (Bremen) $\mathrm{GmbH}$ \\ ${ }^{4}$ Thermo Fisher Scientific \\ Presenting Author: tim.elliott@bristol.ac.uk
}

'Proteus' is a prototype, tribrid mass-spectrometer that couples a magnetic sector, multi-collection system with an inductivelycoupled plasma 'front' end including quadrupole mass-filter and collision cell. This instrument combines the advantages of plasma-ionisation multi-collection with a flexible means to remove troublesome interferences, which have been a limitation to the full application of MC-ICPMS. Here we review the capabilities of this instrument as explored over more than five years of operation in our laboratory.

In the simplest mode of operation, the mass-filter is operated at full transmission and the collision cell simply removes interferences from the spectrum to be analysed. In this way, the efficient charge-transfer between $\mathrm{Ar}^{+}$and $\mathrm{H}_{2}$ enables ${ }^{40} \mathrm{Ca}$ to be measured with precision. We demonstrate the success of such an approach in resolving nucleosynthetic, radiogenic and mass dependent (using a 40-42-43-44 double spike inversion) variations of ${ }^{40} \mathrm{Ca}$ to $\pm 15 \mathrm{ppm}(2 \mathrm{SE}, \mathrm{n}>20)$.

Alternatively, the collision cell can be used to create new analyte species (e.g. $\mathrm{SrF}^{+}$by reaction of $\mathrm{Sr}^{+}$with $\mathrm{SF}_{6}$ and $\mathrm{TiO}^{+}$ by reaction of $\mathrm{Ti}^{+}$with $\mathrm{O}_{2}$ ) which are then chemically resolved from unreactive or less reactive interferences (e.g. ${ }^{87} \mathrm{Rb}^{+}$or ${ }^{50} \mathrm{Cr}^{+}$). Here, the mass-filter is operated in a band-pass mode, such that it transmits only the atomic species of interest into the collision cell and creates a clean background for the mass spectrum of the analysed molecular species. This approach is especially valuable for laser ablation, where there is no opportunity to remove target elements from matrix by prior chemical separation. Thus, we can date in situ common crustal rocks, with minimal sample preparation, to a precision better than $\pm 2 \mathrm{Ma}$ in $400 \mathrm{Ma}$. Further, we can detect, in situ, pre-solar grains in the matrices of chondritic meteorites.

A final arrangement is to run the mass-filter at single mass unit transmission, which in combination with the collision cell and magnetic sector, achieves a dynamic range in isotope ratio measurement of up to $5 \times 10^{-12}$ (e.g. for ${ }^{210} \mathrm{~Pb} /{ }^{208} \mathrm{~Pb}$ ) approaching the performance of accelerator mass-spectrometers. 